\title{
Rapport de la fouille de Demirci-Sinop, 2000
}

Dominique Kassab Tezgör, Fuat Dereli

\section{Citer ce document / Cite this document :}

Kassab Tezgör Dominique, Dereli Fuat. Rapport de la fouille de Demirci-Sinop, 2000. In: Anatolia Antiqua, Tome 9, 2001. pp. 215-225;

doi : https://doi.org/10.3406/anata.2001.969

https://www.persee.fr/doc/anata_1018-1946_2001_num_9_1_969

Fichier pdf généré le 06/04/2018 


\section{RAPPORT DE LA FOUILLE DE DEMİRCİ-SINOP 2000}

La prospection magnétique menée par A. Hesse, M. Drahor et A. Kaya, avait déterminé dans le secteur nord du site de l'atelier amphorique de Demirci, à 15 kilomètres au Sud de Sinop, deux zones principales de concentration de fours ${ }^{1}$. Tandis que la zone $\mathrm{A}$ a été entièrement fouillée ${ }^{2}$, seulement deux anomalies sur quatre de la zone $\mathrm{B}$ ont été dégagées en $1995^{3}$ : un grand bâtiment au sol dallé (a) et un four de taille moyenne (b'), superposé à un autre plus petit $(b)^{4}$.

Pendant la fouille de l'an 2000, les vestiges des deux autrcs anomalies de cette zone ont été mis au jour ${ }^{5}$ (fig. 1). Ainsi que nous l'avions constaté lors de la fouille précédente, l'ensemble de ce secteur a subi un glissement de terrain d'Est en Ouest, de sorte que toutes les structures dégagées ont basculé et sont en pente.

\section{LE FOUR AMPHORIQUE}

L'anomalie située au Sud des fours (b et b') fouillés en 1995 correspond à un autre four en forme de poire (c) (L. : 2,45 m et 1. max. : 1,85 m) (fig. $2-4,9$ et 10). Le mur de la chambre de chauffe, préservé sur toute sa hauteur au Nord-Est (H. : $76 \mathrm{~cm}$ ) est bâti avec trois assises de briques de four surmontées de six autres de tuileaux. La surface supérieure de la dernière arase constitue un redan d'une largeur de $18 \mathrm{~cm}$, au-dessus duquel débute la chambre de cuisson, dont il ne reste que quatre rangs de tuileaux $(\mathrm{H}$. cons. : $14 \mathrm{~cm})$. Les arcades de soutien de la sole partaient de ce redan sur lequel elles prenaient appui, pour aboutir sur les deux piliers centraux qui sont encore debout, également construits en tuileaux (à l'Ouest : H. : $70 \mathrm{~cm}-$ L. : $64-1$. : 28 ; à l'Est : H. : 66 - L. : 68 - 1. : 25). Les parois du four et des piliers sont lutées, et le sol est formé d'une épaisse croûte d'argile cuite posée sur une couche de graviers.

L'alandier de ce four, orienté vers le Sud, est relativement bien conservé. Son entrée, interrompant la chambre de chauffe, est bordée de part et d'autre de deux arcades courbes de terre cuite, surmontées d'un linteau en argile arqué (fig. 5). A l'extérieur du four, il est délimité par deux murs partant obliquement, lui conférant une forme trapézoïdale (L. : 1,22 m-1. : 1,60 m) : celui à l'Ouest comprend trois assises de pierres de rivière et de poudingues, tandis que celui à l'Est n'a conservé qu'une unique pierre volcanique de grande dimension (L. : $1 \mathrm{~m} \mathrm{x} \mathrm{1.} \mathrm{:} 41 \mathrm{~cm} \mathrm{x} \mathrm{H.} \mathrm{:} 56 \mathrm{~cm}$ ) (fig. 6). La mortaise sur la paroi externe de cette dernière

\footnotetext{
*) Université de Bilkent, Département des Beaux-Arts, 06533 Bilkent, Ankara/Turquie.

**)Musée archéologique, Sinop/Turquie.

1) Drahor, Hesse et Kaya, 1995

2) Kassab Tezgör, 1996; Kassab Tezgör et Tatlıcan, 1998.

3) Kassab Tezgör et Tatlican, $1996: 344-354$.

4) Afin de recaler plus sûrement le carroyage de la fouille 2000 sur celui de la fouille 1995 et de faciliter le tracé du plan masse de la zone, nous avons dégagé de nouveau le mur nord du bâtiment (a) ainsi que l'arrière des deux fours (b et b').

5) Ont participé à cette fouille Jean-François Bernard, architecte (IFEA), assisté par Elif Kandir, architecte (assistante à l'Université Mimar Sinan d'Istanbul) et par Hamza Kayıcı, topographe (cadastre de Sinop), Semay Taneri, ingénieur, Nilay Karakaş, restauratrice, Seyda Sever, photographe (assistante, Université d' $\Lambda$ nkara), Senem Ozden, étudiante (Université d'Istanbul), ct Evim Güvem, étudiante (Université d'Ankara). Outre le financement de la Commission des Fouilles, les travaux ont été parrainés par les Caves des Vins Kavaklıdere comme chaque année et nous en remercions vivement Monsieur Başman.
} 


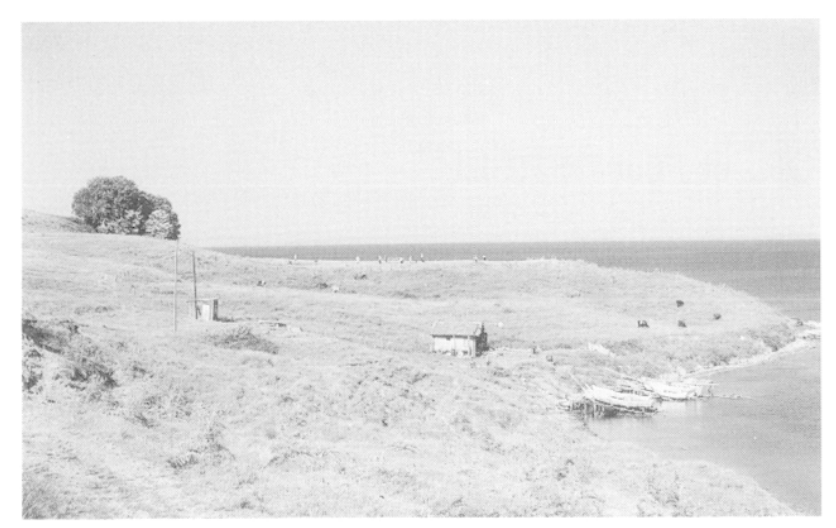

Fig. 1 : Vue de la zone $B$ pendant les fouilles. montre qu'il s'agit d'un réemploi. Cette pierre n'est pas sans rappeler les pierres dites de devantelle que l'on a retrouvées à l'entrée du four b' et d'un four de la zone A, dont l'alandier a également une forme trapézoïdale ${ }^{6}$.

Lorsque ce four a été mis au jour, il était comblé essentiellement de tuileaux et de tubulures à pâte claire, caractérisées par un col conique et fermé ${ }^{7}$ (fig. 7). A près de dix reprises, elles ont été retrouvées enfilées les unes dans les autres et maintenues ensemble par de la pâte argileuse cuite, formant un assemblage de forme courbe probablement utilisé pour la construction de la couverture de la chambre de cuisson (fig. 8). Etaient mêlés à ces objets un grand nombre de supports, dont cer-

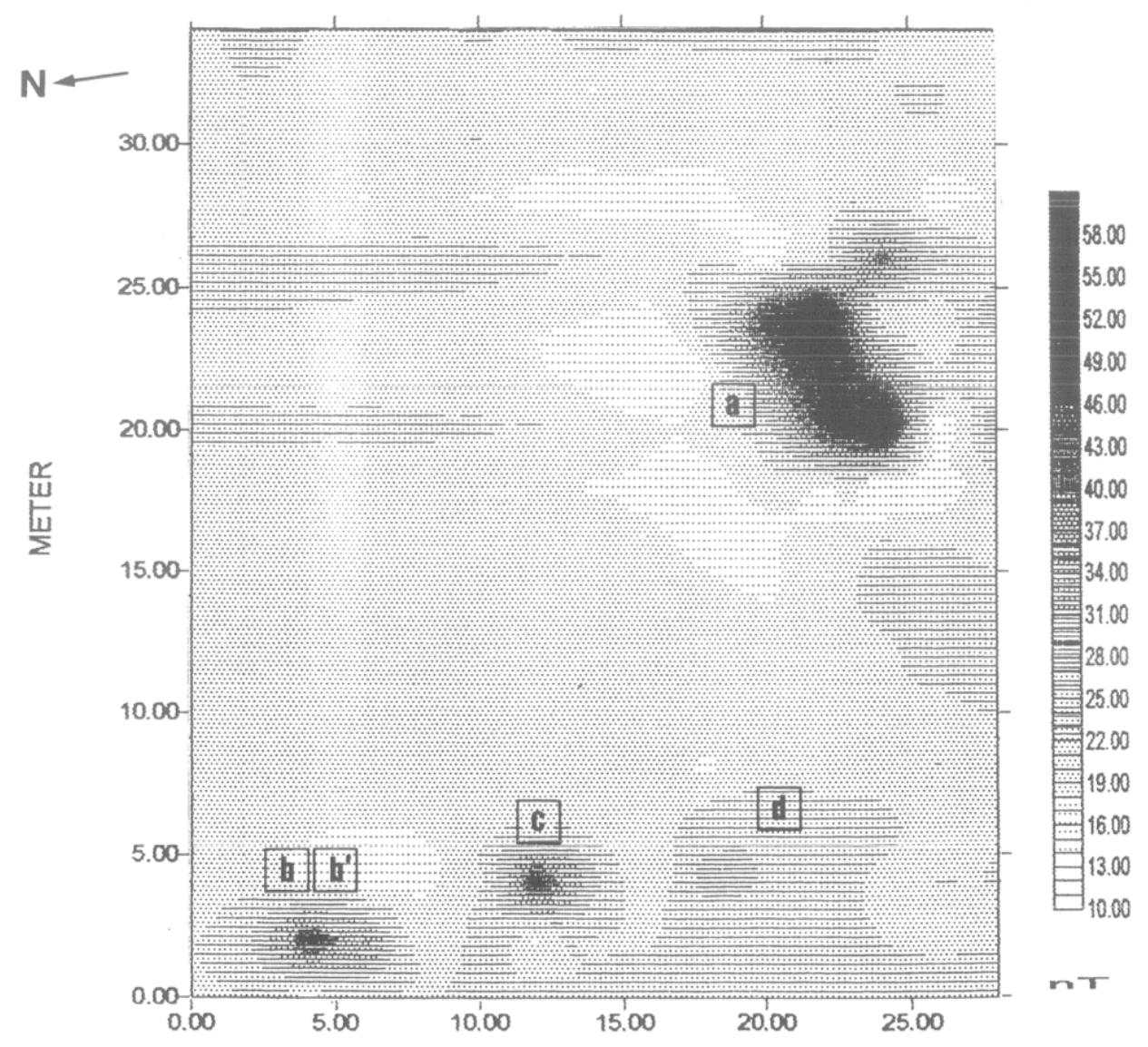

METER

Fig. 2 : Carte magnétique de la zone B (A. Hesse).

6) Kassab Tezgör, $1996: 353$ et Kassab Tezgör et Tatlıcan, $1998:$ 429-432, fig. 11, 12 et 16.

7) Kassab Tezgör et Tatlıcan, $1998: 433$, fig. 18 et 19. 


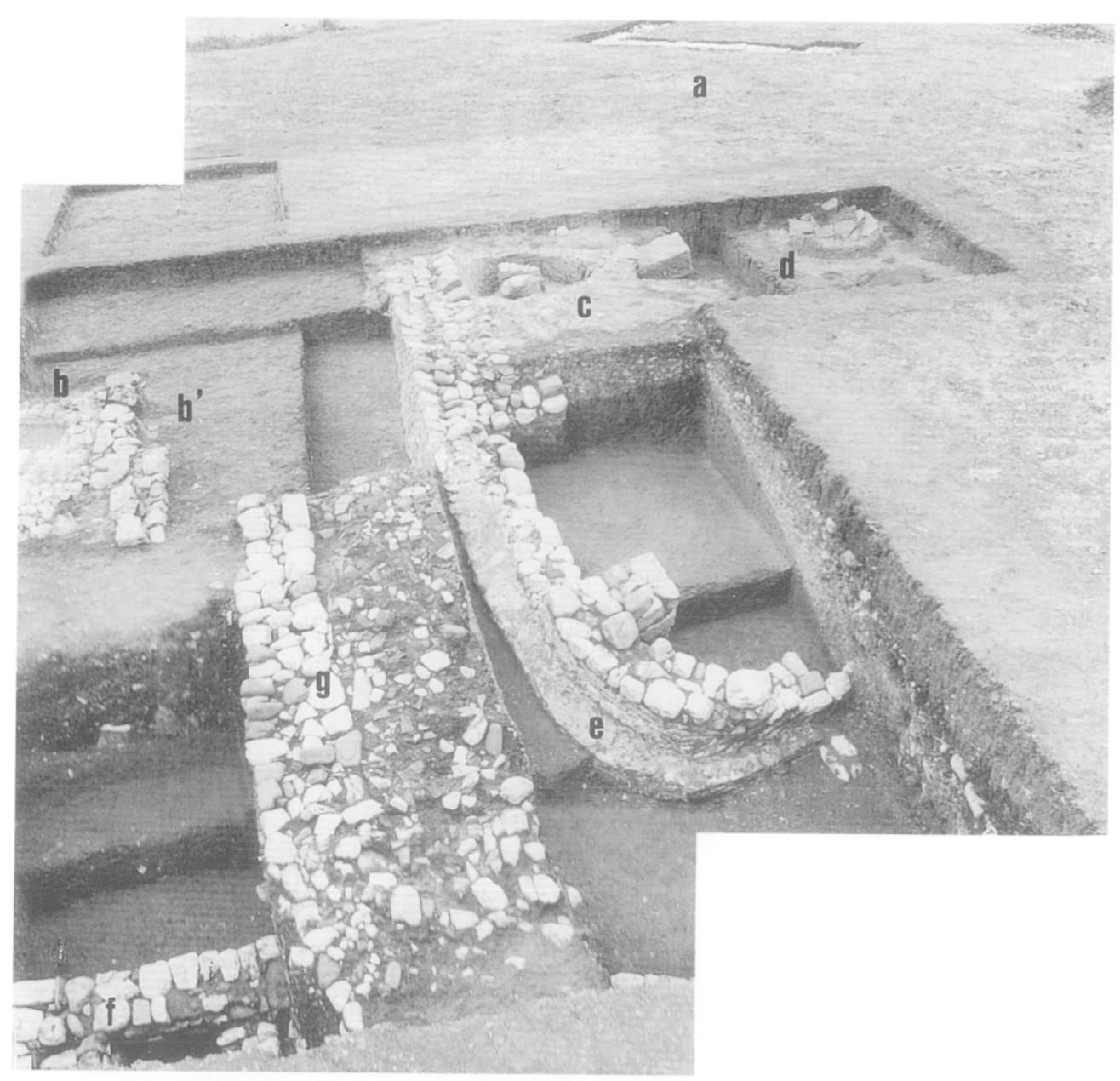

Fig. 3 : Montage photographique de l'ensemble de la zone B (Ş. Sever).

tains inscrits, ainsi que des fragments d'amphores à pâte claire et à fond convexe, d'un type bien connu par les fouilles des années précédentes ${ }^{8}$ (fig. 17 f). C'est un remplissage du même type qui avait été retrouvé dans le four b' et dans celui de la zone $A$ mentionné plus haut, à la différence qu'il s'agissait d'amphores à pied cylindrique, d'une morphologie et d'une technique de fabrication semblables sinon en tous points ${ }^{9}$ (fig. $17 \mathrm{~g}$ ).

\section{PITHOI AU SUD DU FOUR}

De faible densité, l'anomalie la plus au Sud (d) ne signalait que deux pithoi (fig. 2, 3, 9 et 10). Ils ne sont conservés que jusqu'à hauteur de leur plus grand diamètre. Il est difficile de décider à quel stade d'occupation du site ils appartiennent. Toutefois, il est possible qu'ils soient reliés à ce four, car nous avons rencontré à plusieurs reprises des pithoi à proximité d'un four ${ }^{10}$.

Comme il est habituel, ils ont été comblés par un matériel abondant et varié : céramiques communes, céramiques à feu, lampes (fig. 12), et bien sûr amphores. Signalons dans le pithos ouest le haut d'un exemplaire d'un type à pâte claire produit à Demirci, mais jamais encore rencontré aussi complet sur le site (fig. 11 et 17 e).

8) Kassab Tezgör, sous presse.

9) Ibidem ; Kassab Tezgör, 1996 : 354 ; Kassab Tezgör et Tatlıcan, $1998: 433$ sq., fig. 20

10 Kassab Tezgör, 1996 : 342 sq., fig. 15 ; Kassab Tezgör et Tatlıcan, 1998 : p. 430, fig. 11 (à l'Ouest du petit four), p. 438 et p. 440 , fig. 31 . 


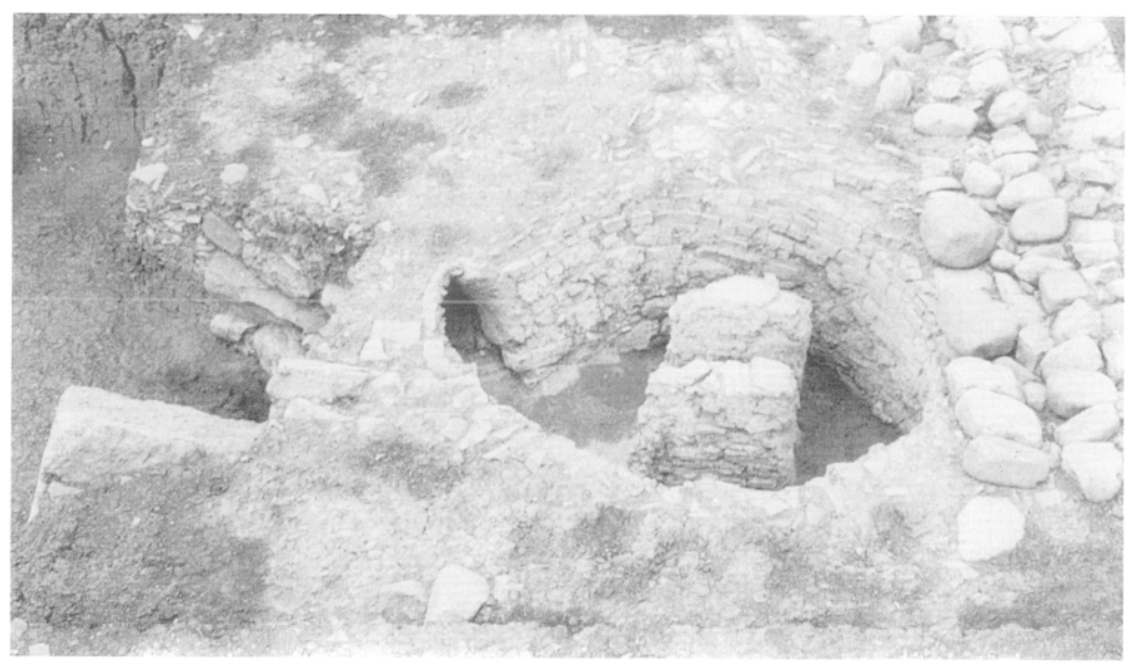

Fig. 4 : Vue du four depuis I'Est.

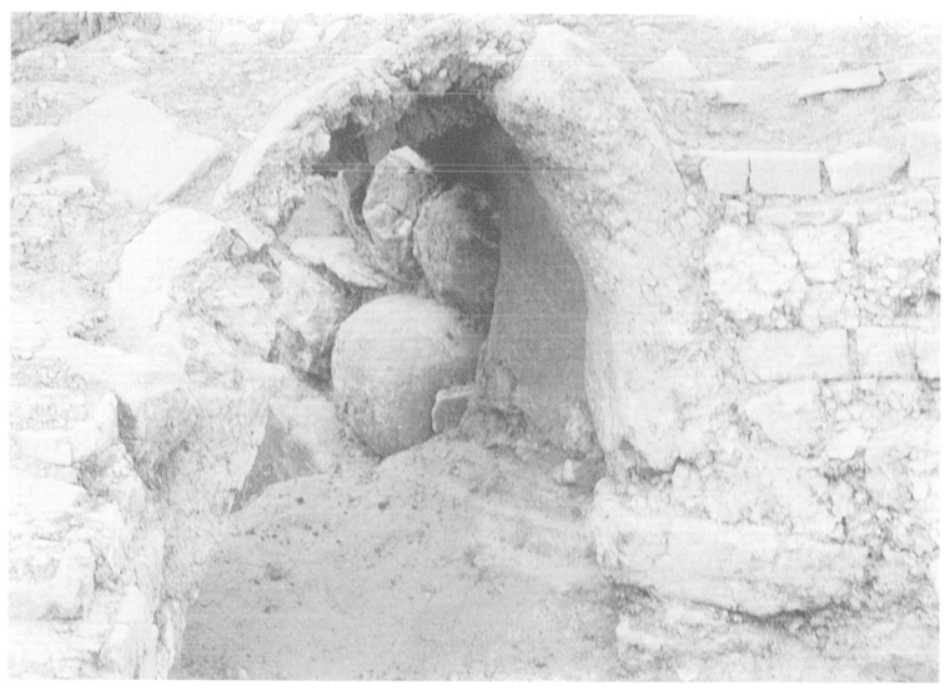

Fig. 5 : Vue des arcades de soutien de l'alandier.

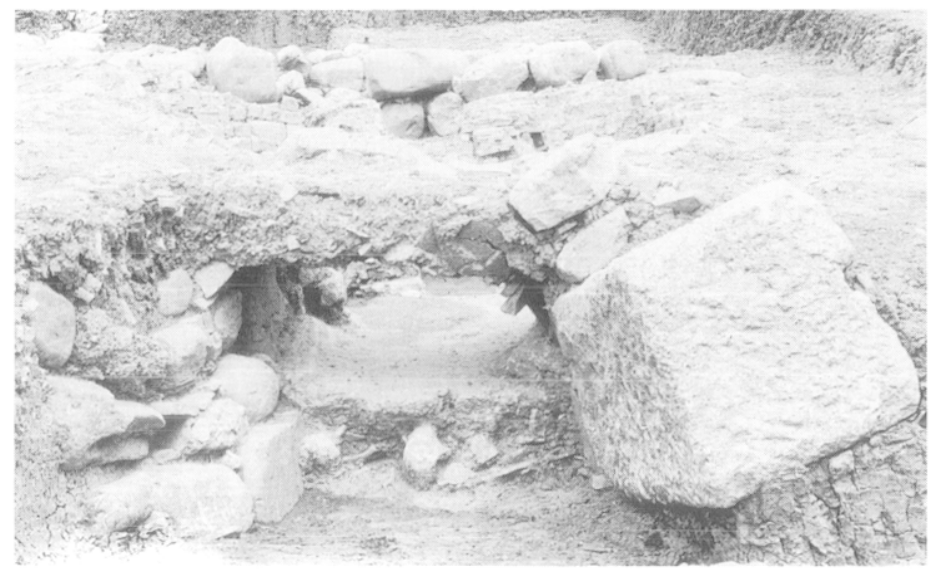

Fig. 6 : Vue de l'alandier. 


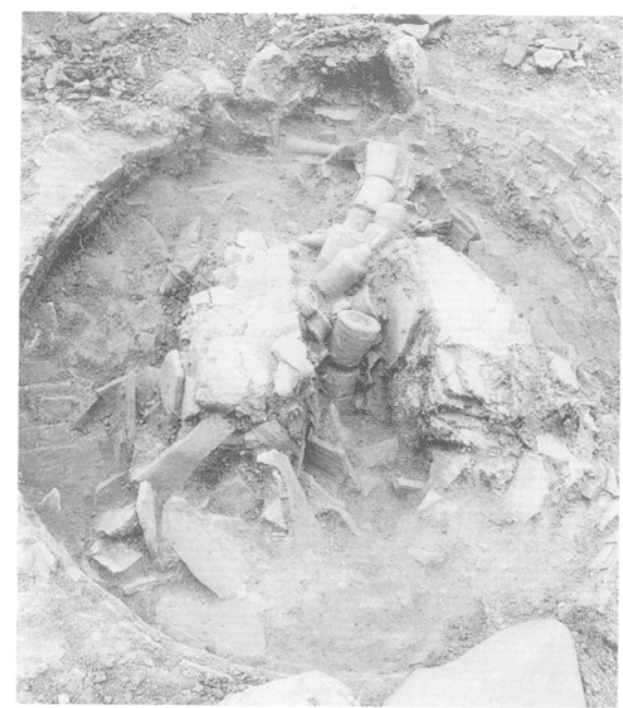

Fig. 7 : Remblayage du four avec tubulures et tuileaux.

\section{FOUILLES DES STRUCTURES A L'OUEST DE LA CARTE MAGNETIQUE}

Nous avons été amenés à étendre la fouille à l'Ouest de la carte magnétique, afin de dégager les constructions apparues dans les carrés déjà ouverts.

\subsection{Bâtiment en abside}

La chambre de cuisson du four c a été détruite et le four remblayé pour permettre la construction d'un bâtiment orienté vers l'Ouest, où il se termine en abside (e) (fig. 3, 9, 10 et 13).

Il est entièrement doublé à l'extérieur par un mur de sept assises de pierres et de tuiles en alternance, liées par du ciment, la dernière assise de pierres étant elle-même recouverte d'une couche de ciment visible le long de l'abside (H. max. : $104 \mathrm{~cm}$ - 1. max. : $52 \mathrm{~cm}$ ). Ce mur devait être enfoui dans les fondations et servir de contrefort à la construction.

Le mur latéral du bâtiment étant conservé jusqu'à la même hauteur que le mur cimenté, seul son parement intérieur est visible avec trois assises. La partie nord de l'abside, en revanche, est mieux préservée, avec à partir du contrefort une première assise de pierres, surmontée de trois assises de tuiles, auxquelles succède une autre assise de

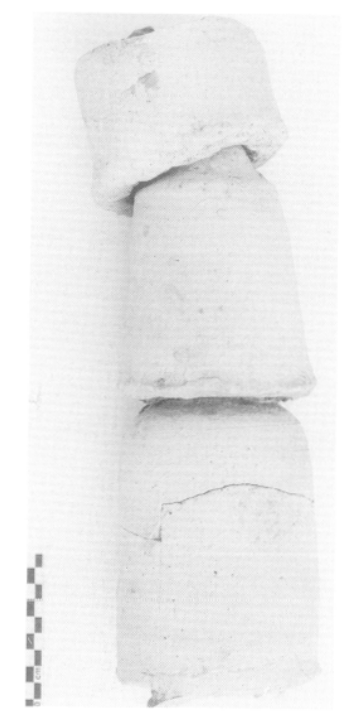

Fig. 8 : Tubulures enfilées les unes dans les autres (Ş. Sever).

pierres. La construction, qui semble être un pilier accolé à la paroi interne de l'abside, est postérieure.

Notons que le remblai à l'Ouest de l'abside renfermait une poche de fragments d'amphores à pâte rouge ${ }^{I 1}$. Ce sont généralement des cols, avec ou sans anses, qui appartiennent essentiellement au type en carotte : ils sont hauts et étroits, terminés par une lèvre en double bourrelet ou en méplat (fig. 16 et $17 \mathrm{~d}$ ). D'autres se rattachent au type intermédiaire, moins hauts au-dessus des anses et légèrement plus larges (fig. 15 et $17 \mathrm{c}$ ). Plus rares sont ceux qui correspondent aux types amphoriques les plus volumineux : ils peuvent être larges et courts, bordés d'une lèvre en bourrelet haut et plat (fig. 14 et $17 \mathrm{a}$ ), ou encore larges et hauts, avec une lèvre en double bourrelet (fig. $17 \mathrm{~b}$ ).

Ce bâtiment, dont la longueur maximale dégagée est de $9,50 \mathrm{~m}$, ne semble pas avoir été préservé à l'Est du four. En revanche, l'abside se poursuit sans doute au-delà de la limite sud de la fouille, bien qu'ayant subi des éboulements.

\subsection{Structures à l'Ouest des fours fouillés en 1995}

Des murs à trois assises (f) ont été mis au jour à l'Ouest des fours fouillés en 1995, profondément enfouis en raison du mouvement de terrain d'Est en 


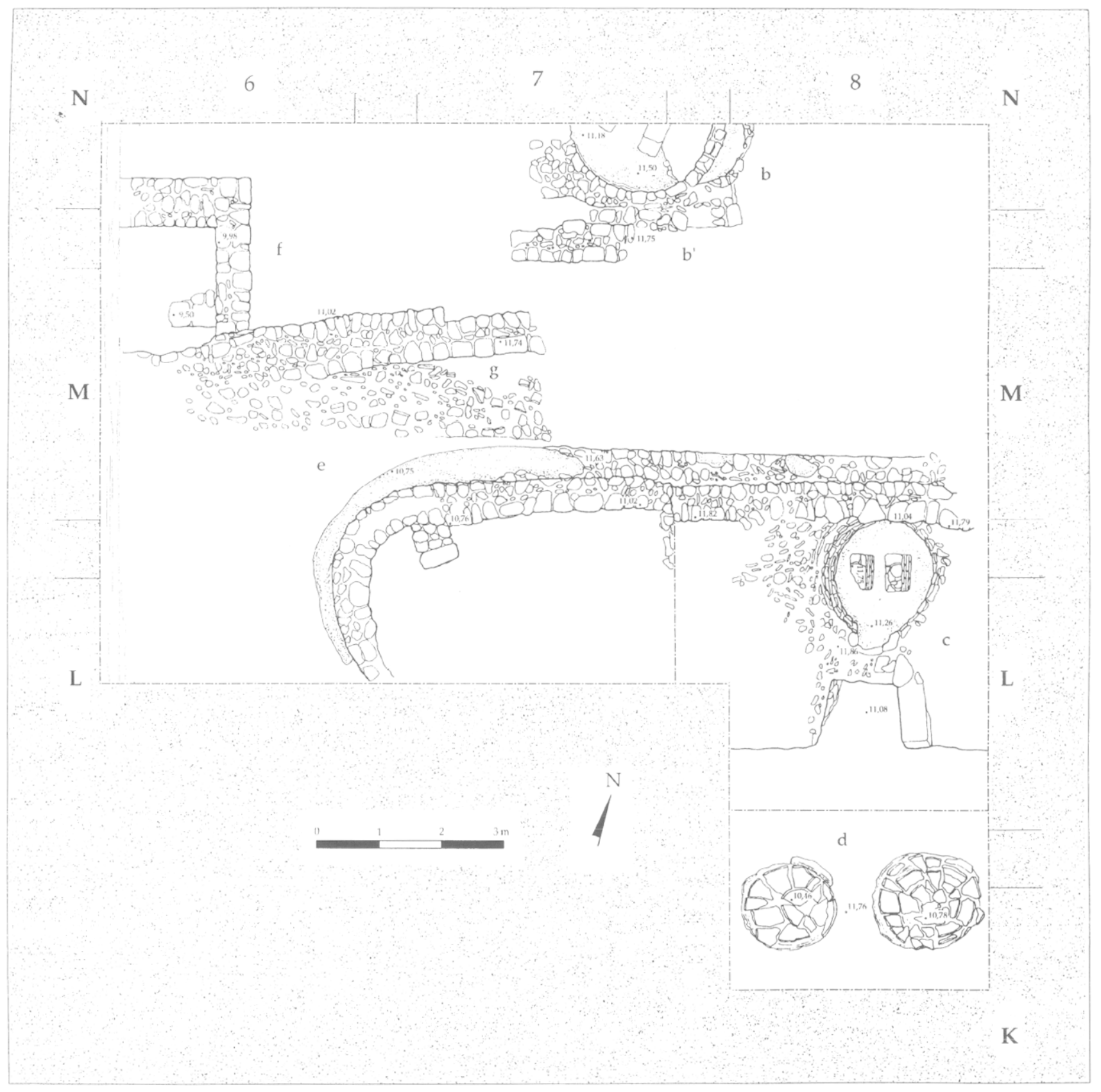

Fig. 9 : Plan de l'ensemble de la fouille 2000 (J.-F. Bernard). 


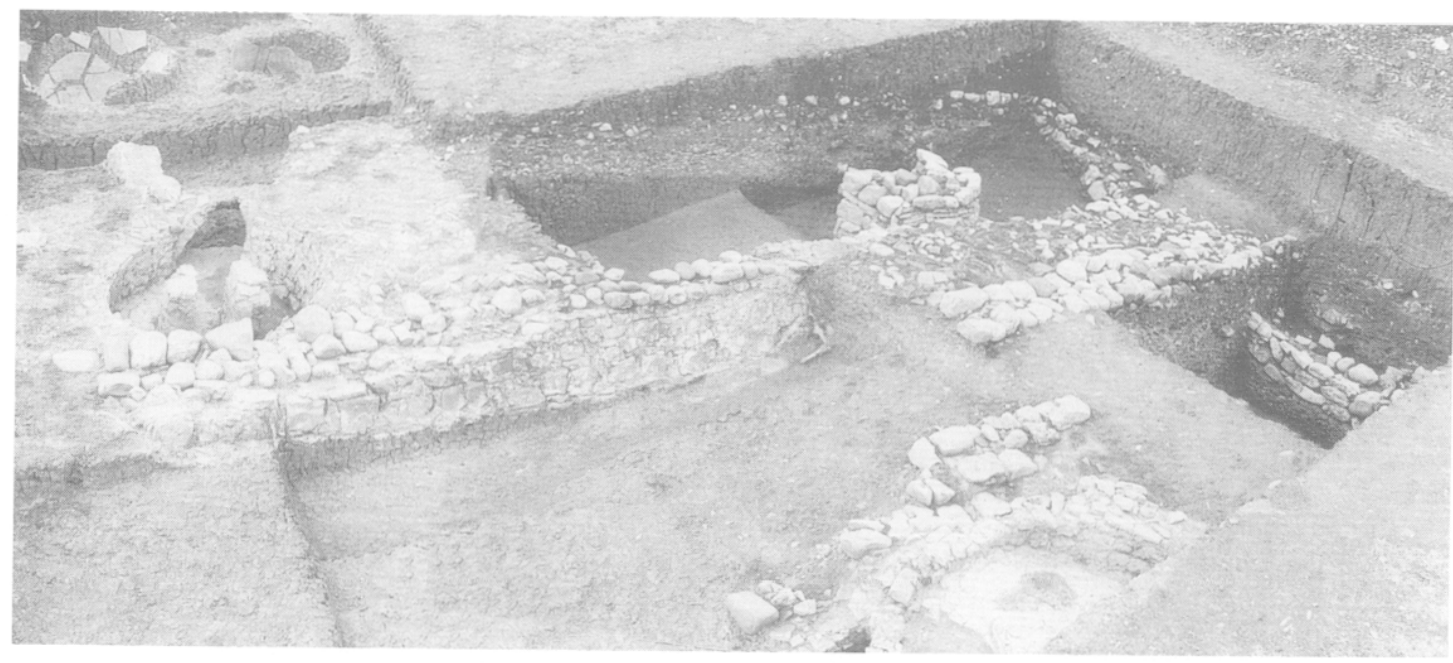

Fig. 10 : Montage photographique de l'ensemble de la fouille (Ş. Sever).

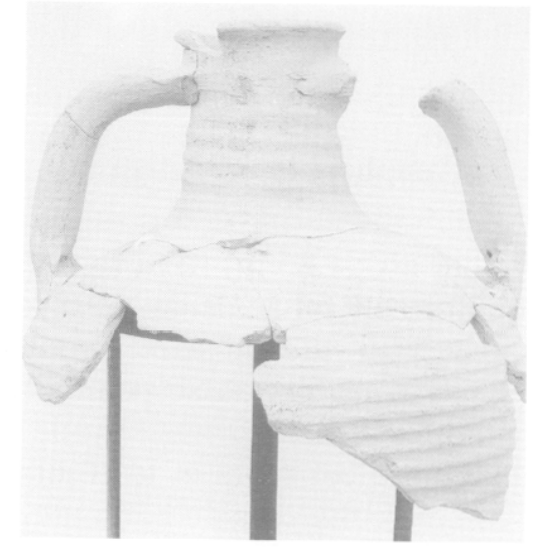

Fig. 11 : Amphore à pâte claire (restauration N. Karakaş) (S Sever).

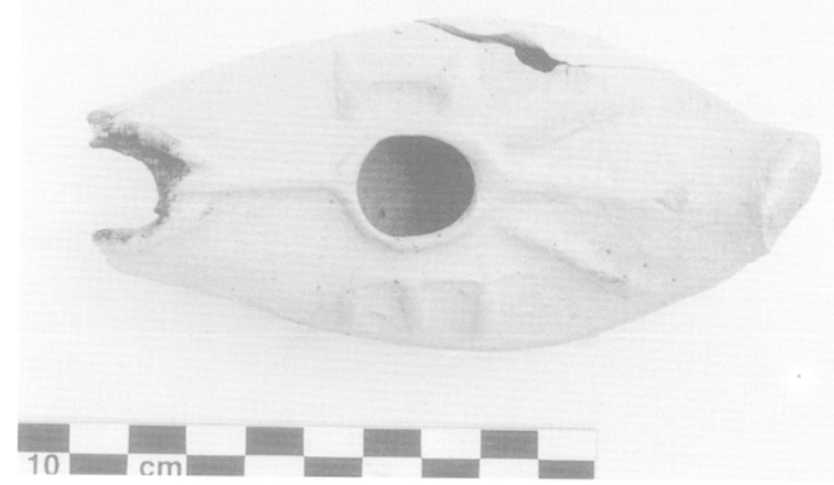

Fig. 12 : Lampe (Ş. Sever).

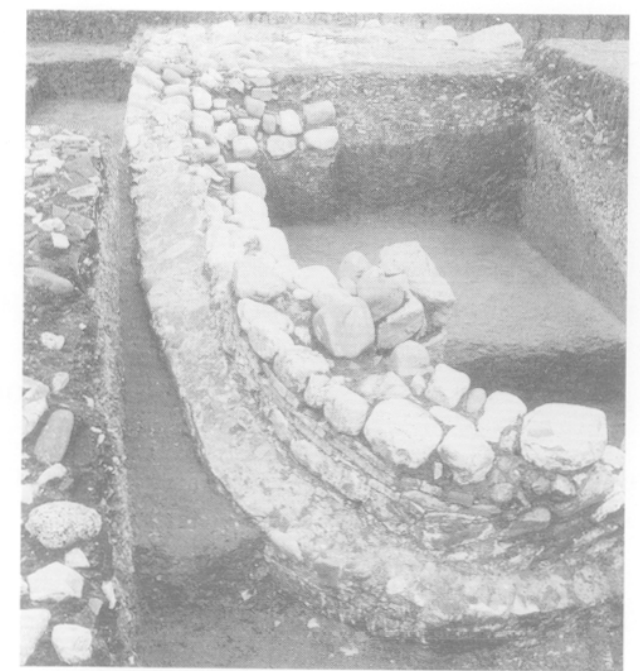

Fig. 13 : Vue du bâtiment avec l'abside depuis l'Ouest (Ș. Sever). 


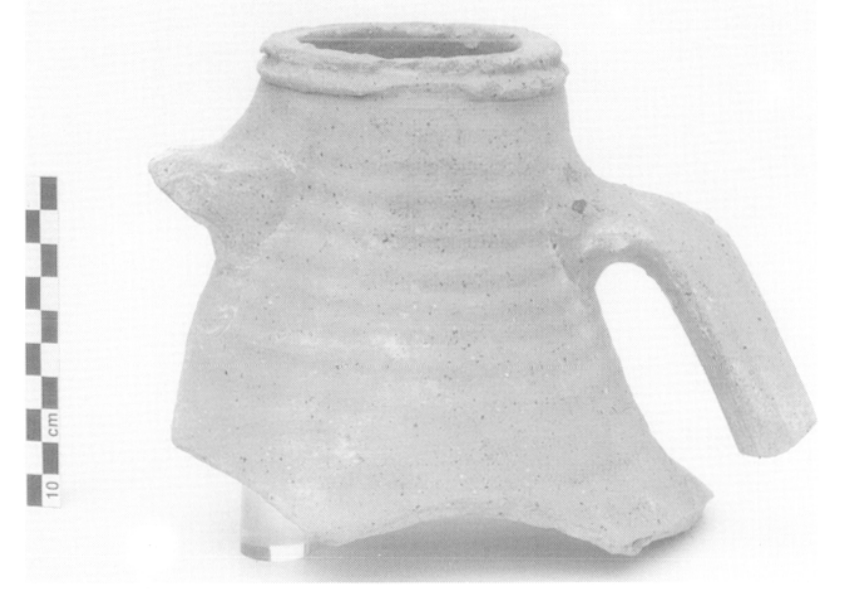

Fig. 14 : Col d'amphore (Ş. Sever).

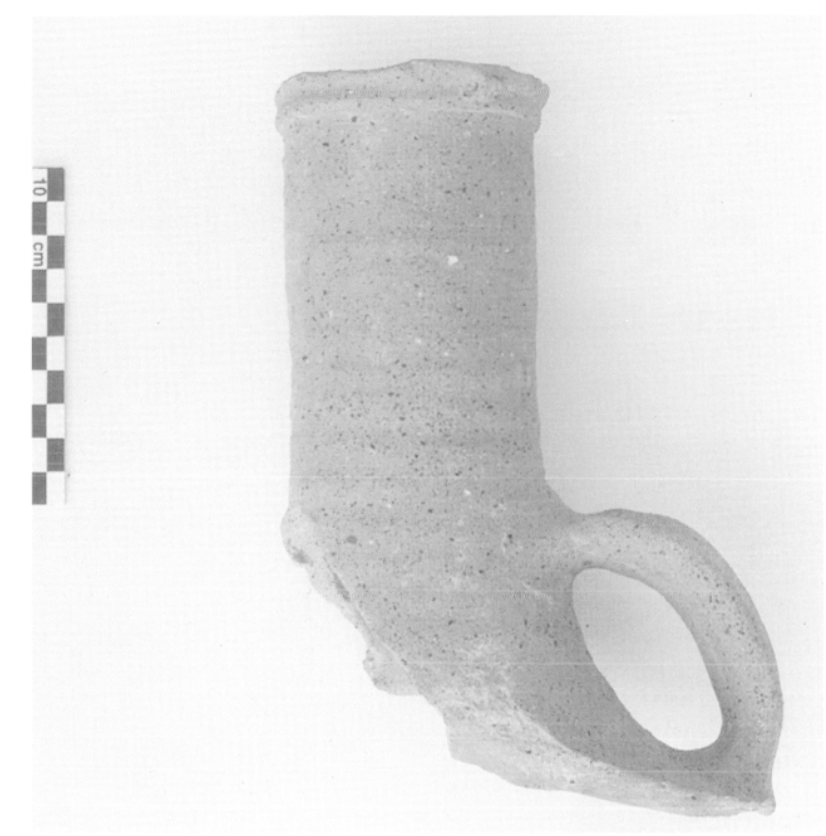

Fig. 16 : Col d'amphore carotte (Ş. Sever).

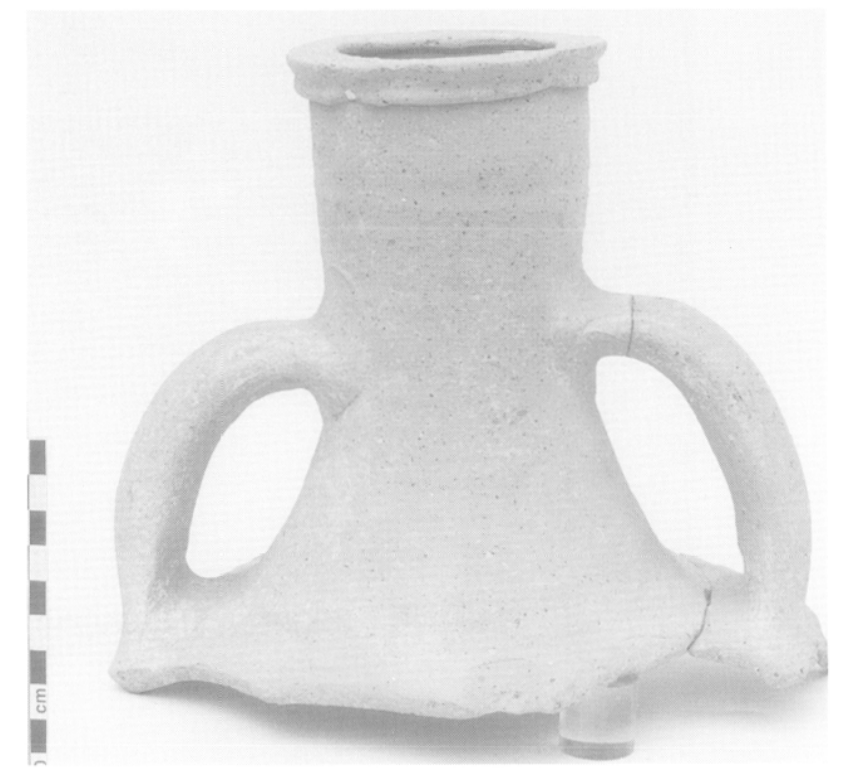

Fig. 15 : Col d'amphore intermédiaire (Ş. Sever).

Ouest (L. du mur est : 1,85 m) (fig. 3, 9 et 10). Les deux murs qui forment un angle au Nord sont châ̂nés, tandis que celui au Sud a été ajouté postérieurement. Cette construction se poursuit au-delà des limites de la fouille.

Le remblai entre ces murs et les fours b et b' comprenait comme celui près du bâtiment à abside une poche d'amphores à pâte rouge. Il est à mettre en relation avec la couche de tessons fouillée en 1995 au Sud des alignements superposés de cols et de panses qui partaient du four $b^{12}$.

Entre ces murs et l'abside a été dégagé un alignement de pierres sur une assise (f) (fig. 3, 9 et 10). Il est jouxté de tuiles et de pierres éparpillées avec des traces d'incendie, qui semblent provenir de l'effondrement d'une toiture ${ }^{13}$.

\section{CHRONOLOGIE RELATIVE DES STRUCTURES DE LA ZONE B}

La zone B comprend donc dans son ensemble trois fours (b, b' et c), dont deux superposés, deux bâtiments de grande taille : celui terminé en abside

12) Kassab Tezgör, $1996: 352$, fig. 30 et p. 353.

13) Les pierres qui apparaissaient à la limite sud de la fouille 1995 et que nous avions pensé appartenir à un autre four font partie de cet ensemble : Kassab Tezgör, $1996: 354$ et p. 352, fig. 30. 

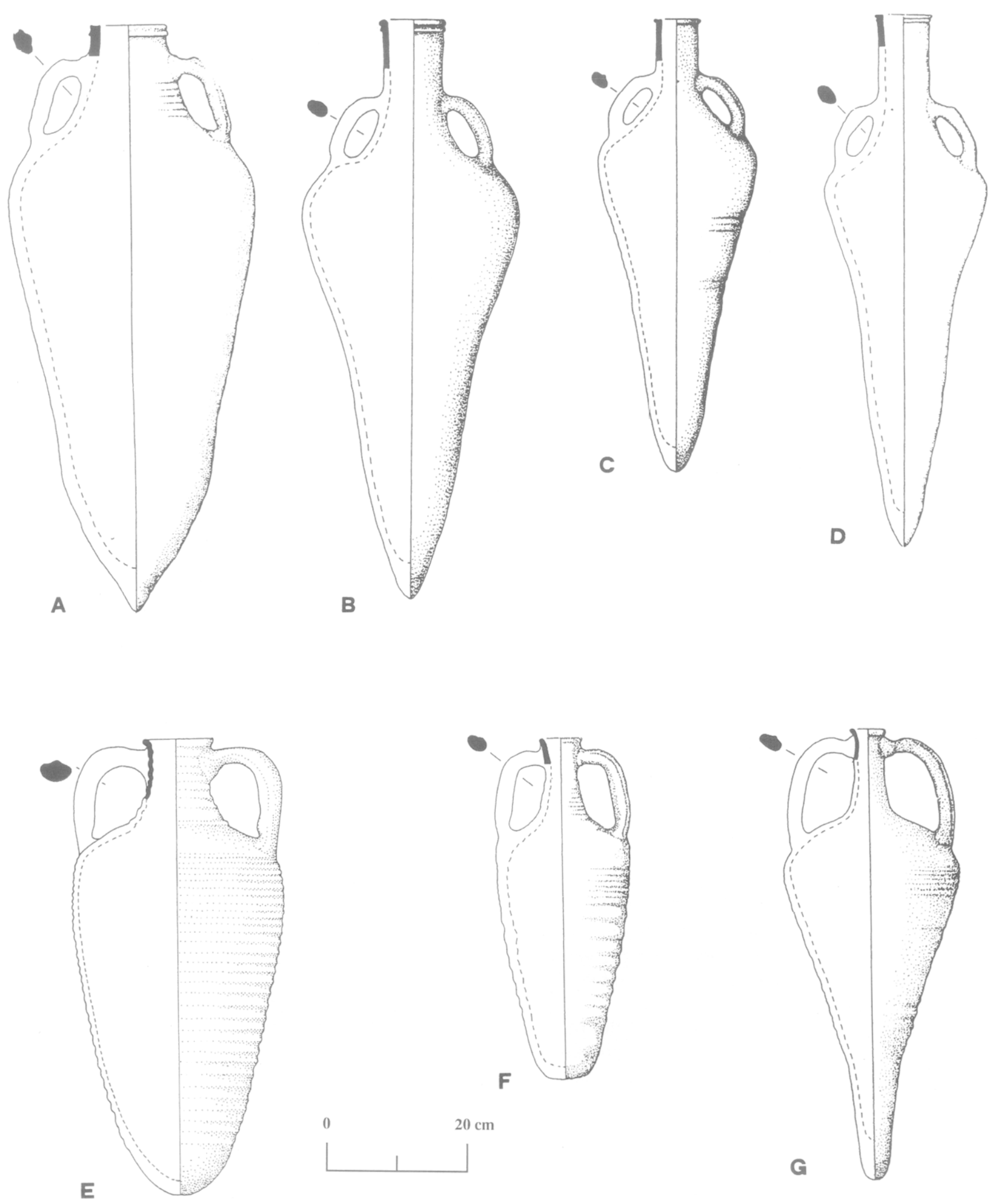

Fig. 17 : Répertoire des formes mentionnées produites à Demirci (A. Özkan). 


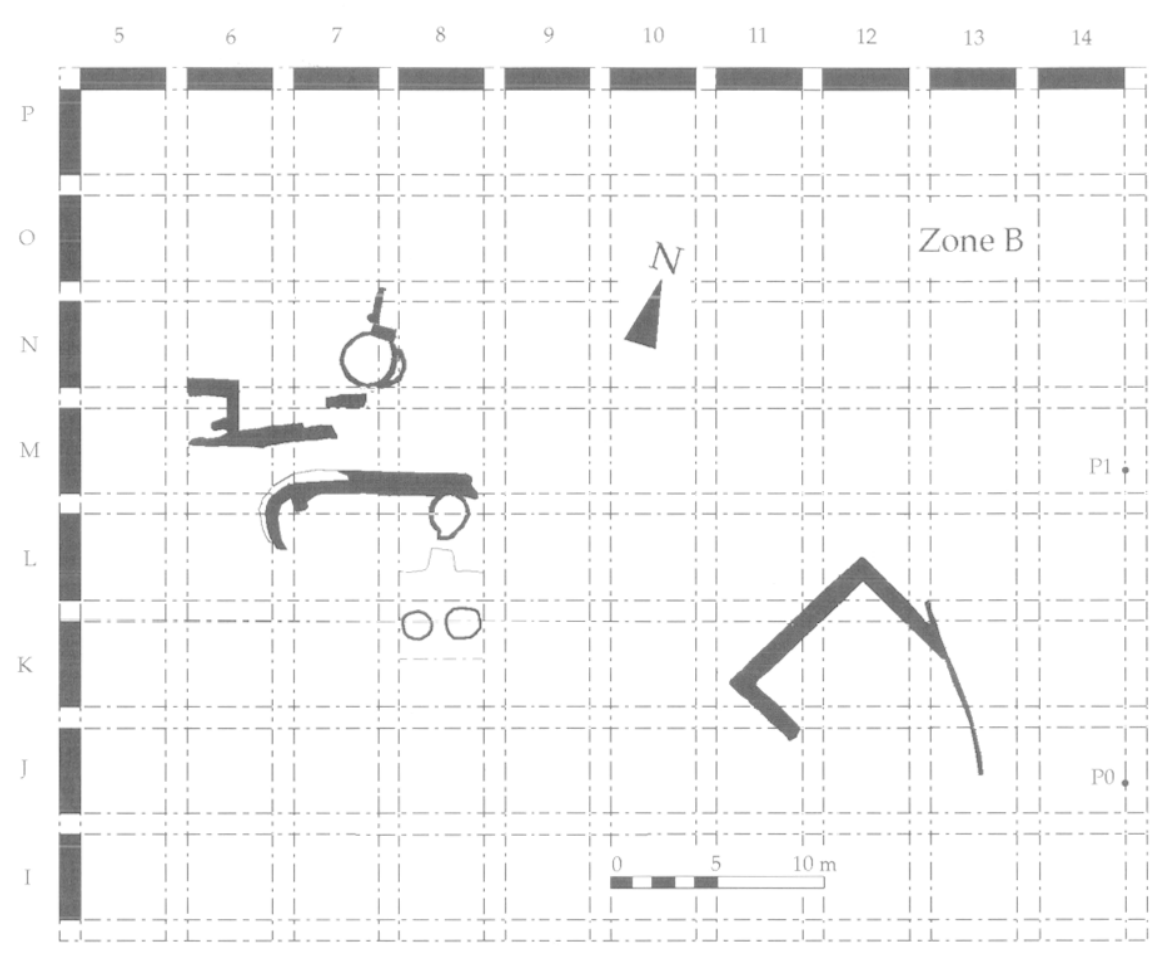

Fig. 18 : Plan masse de la zone B (J.-F. Bernard).

(e) et celui au sol dallé (a), un autre dont seulement un angle a été dégagé (f), et enfin les vestiges d'une construction sans doute effondrée accidentellement (g) (fig. 3 et 18).

Il semble que nous puissions établir pour la zone $\mathrm{B}$ une séquence chronologique avec deux activités différentes qui se sont succédées. Ce secteur avait connu en outre une première occupation à l'âge du Bronze, comme l'avait montré une couche de céramiques au-dessous du bâtiment dallé $(a)^{14}$.

\subsection{Le site aménagé comme atelier}

- Le petit four fouillé en 1995 (b) est la plus ancienne structure de cette zone. Pour permettre la construction du four suivant, il a été comblé d'amphores à pâte rouge.

- Le four qui le chevauche (b') et celui plus au Sud fouillé cette année (c) sont contemporains. Le remblayage de ces deux fours consiste en amphores à pâte claire, et l'on dispose pour celui du four b' d'un terminus post quem avec deux monnaies de Justin (565-578 ap. J.-C.) ${ }^{15}$.

\subsection{Le site aménagé comme espace public}

Le bâtiment en abside (e) qui surmonte le four (c) est par conséquent contemporain ou postérieur à Justin et se situe au plus tôt dans la seconde moitié du VI ${ }^{e}$ s. ap. J.-C. Il est possible que les murs chaînés (f) soient de la même date, ainsi que le grand bâtiment dallé (a) de 1995.

- La construction effondrée (g) est postérieure, car construite au-dessus du bâtiment en abside et des murs chaînés.

Le glissement de terrain peut être la raison de l'abandon de ce secteur du site, mais nous ne disposons pas d'indice qui permette de le situer dans le temps ${ }^{16}$.

14) Kassab Tezgör, $1996: 346$.

15) Kassab Tezgör, $1996: 354$.

16) Deux mouvements de terrain ont également affecté la région de Çiftlik, éloigné de cinq kilomètres au Nord de Demirci. Le dernier d'entre eux a provoqué l'abandon de la basilique alors qu'elle était encore inachevée (Hill, $1998: 297$ sq.). Cette catastrophe, probablement survenue à la fin du IV ${ }^{\mathfrak{e}}$ s. ap. J.-C., est antérieure à celle observée dans la zone $\mathrm{B}$ de Demirci. Il faut noter que la zone A du site n'a pas du tout été touchée. 
Aucune des structures dégagées ne peut recevoir une identification. Elles sont toutefois probablement à inscrire dans le cadre d'un nouvel aménagement du site après l'arrêt de l'activité céramique. Les dimensions des constructions et la simplicité de leur plan laissent plutôt penser à des lieux d'utilité publique. Ainsi, l'histoire de l'occupation de la zone $\mathrm{B}$ ne se limite pas à la production amphorique : c'était également le cas dans la zone $A$, où l'atelier a laissé place, au moins en partie dès le VII ${ }^{\mathrm{e}}$ s. ap. J.-C., à des pressoirs de vin ou d'hui$\mathrm{le}^{17}$.

D.K.T. et F. D.

\section{BIBLIOGRAPHIE}

Drahor, M.G., Hesse, A. et Kaya, M. A., 1995 : "Prospection magnétique des ateliers amphoriques de Sinop", Jeofizik 9, p. 7-12 (en turc).

Hill, S., 1998 : "Rescue Excavations at Çiftlik (Sinop)", dans R. Matthews (éd.), Ancient Anatolia, p. 285-300.

Kassab Tezgör, D., 1996 :"Fouilles des ateliers d'amphores à Demirci près de Sinop en 1994 et 1995", Anatolia Antiqua IV, p. 335-354.

Kassab Tezgör, D., et Tatlıcan, 1., 1998 : "Fouilles des ateliers d'amphores à Demirci près de Sinop en 1996 et 1997", Anatolia Antiqua VI, p. 423-442.

Kassab Tezgör, D., sous presse : "La production d'amphores à Sinope et ses relations avec la Colchide", Actes du 9ème Symposium International, Vani, Géorgie, 1999. 